\title{
Lift on side by side intruders of various geometries within a granular flow
}

\author{
M. F. Acevedo-Escalante ${ }^{1,{ }^{*}}$ and G. A. Caballero-Robledo ${ }^{1}$ \\ ${ }^{1}$ CINVESTAV-Monterrey, PIIT, Nuevo Leon, 66600, Mexico
}

\begin{abstract}
Obstacles within fluids have been widely used in engineering and in physics to study hydrodynamic interactions. In granular matter, objects within a granular flow have helped to understand fundamental features of drag and lift forces. In our group, we have studied numerically the flow mediated interaction between two static disks within a vertical granular flow in a two-dimensional container where the flow velocity and the distance between obstacles were varied. Attractive and repulsive forces were found depending on flow velocity and separation between intruders. The simulations evidenced a relationship between the average flow velocity in a specific section ahead of the obstacles and the attractiverepulsive lift. On the other hand, it was showed that the lift force on an object dragged within a granular medium depends on the shape of the intruder. Here we present experimental results of the interaction between two side-by-side intruders of different shapes within a vertical granular flow. We built a quasi-twodimensional container in which we placed the intruders and using load cells we measured lift and drag forces during the discharge process for different flow velocities.
\end{abstract}

\section{Introduction}

The interaction of obstacles within a flow is widely studied for theory and applications in fluid mechanics. In granular matter, similar experiments where obstacles are immersed in a granular medium, have been made because they are useful to understand mechanical properties in this area.

Granular matter community has thoroughly studied forces on objects immersed in granular flows [3-6]. Flow velocity and its fluctuations have shown to play an important role in attractive and repulsive regimes in flow mediated interactions between obstacles, and lift forces have been associated with a Bernoulli-like phenomenon: a pressure drop associated to flow velocity.

On the other hand, lift forces have been shown to depend on the shape of the immersed object which is due to the "asymmetry in pressure caused by gravity between the lower and the upper parts of the object" [2].

Recently, it was shown numerically that in a static pair of obstacles within a granular flow exists attractive and repulsive lift forces depending of the flow velocity and the separation between them [1]. Among other things, it is found that the mechanism to explain these interacting forces is not a Bernoulli-like effect.

In the present work we show the experimental results for drag and lift forces on obstacles of different shapes placed into a gravitational granular flow.

\section{Experimental Setup}

The experimental setup consists of a vessel built with two $125 \times 50 \times 1.3 \mathrm{~cm}^{3}$ polycarbonate sheets separated by a gap of $1.3 \mathrm{~cm}$ (Fig. 1a and Fig. 1b).

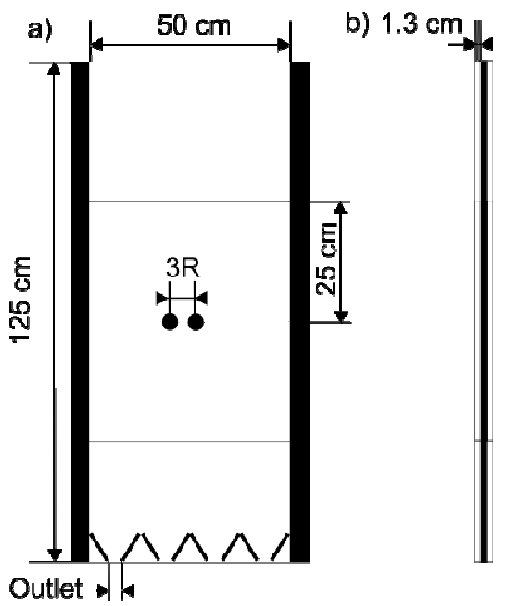

c)
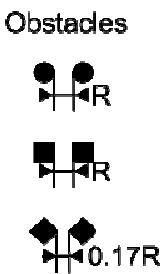

14

$-42 R$

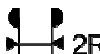

$\mathrm{R}=0.75 \mathrm{~cm}$

Fig. 1. Experimental Setup. A) Experimental Setup front View. B) Experimental Setup Side View. C) Pair of Obstacles used.

The vessel is filled with $6 \mathrm{~kg}$ of tapioca seeds of $3.3 \pm$ $0.2 \mathrm{~mm}$ in diameter using a hopper placed at the top of the vessel. The gap between the sheets is approximately four times the size of the seeds.

The mass flow is controlled by a system of outlets of variable area placed at the bottom of the container. The 
area of the outlets is controlled by varying the angle of the sticks that form the outlets, as can be seen in Figure 1a. The mass flow was measured by collecting the mass flowing through the bottom outlets in a container placed on a scale.

At a height of $55 \mathrm{~cm}$ from the bottom of the vessel a pair of obstacles are connected to a pair of uncoupled load cells of $780 \mathrm{~g}$ capacity which measure drag and lift forces. The pairs of obstacles that were used in the experiments are shown in Fig. 1c: cylinders of radius R; squares of side R placed horizontally and diagonally; and semicircles with the round side facing the centre and towards the walls. The experimental procedure used to measure the forces was as follows: A strip is placed on the bottom of the container to prevent flow. The data logger is initialized and by filling from the top, the container is completely filled. After about 10 seconds the base strip is removed and the system begins to flow. When the system is emptied, data collection is completed. This procedure is repeated for different mass flows and for each pair of obstacles. Finally, particles velocity was measured using a high speed camera to film the system flowing free of obstacles and analysed with a Particle Image Velocimetry (PIV) algorithm using the open source software ImageJ. This software consists in identify the positions of the particles in two sequential images and determine the mean velocity of the particles.

\section{Experimental Results}

\subsection{Mass Flow}

Figure 2 shows the results obtained for all configurations of pairs of obstacles. First, this plot shows that the mass flow is similar in all the outlets used in these experiments and, second, the plot is not linear. This last result was expected because it is consistent with Beverloo's Law for the relation between the quantity of particles falling through an outlet and outlet's area [8].

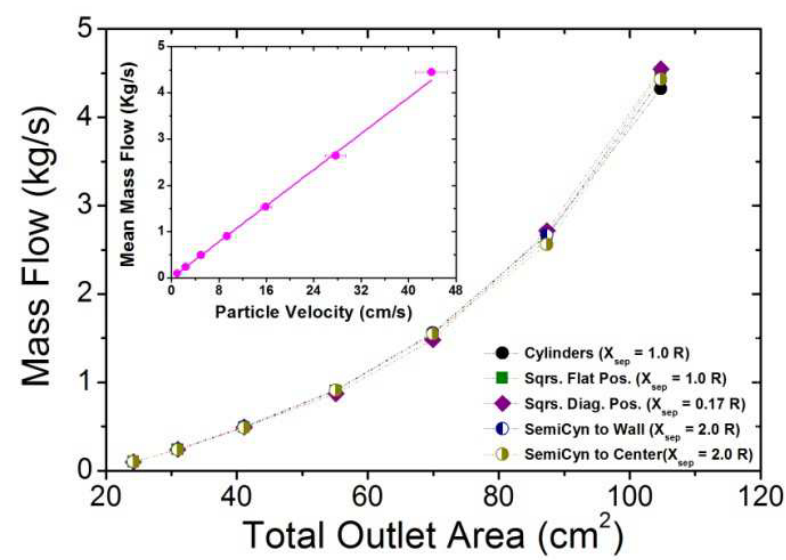

Fig. 2. Mass Flow as a function of total outlet area. Inset: The mean mass flow as a function of particle velocity obtained by PIV.

Our PIV analysis could be tested by plotting the velocity measured by this method and the mass flow directly measured with the scale at the outlet. The relation between these two quantities is linear and is shown in the inset of the Fig. 2.

\subsection{Drag Force}

In this study we define Drag force as the force in vertical direction perpendicular to the direction of the flow. In Fig. 3 Drag force as a function of flow velocity is plotted.

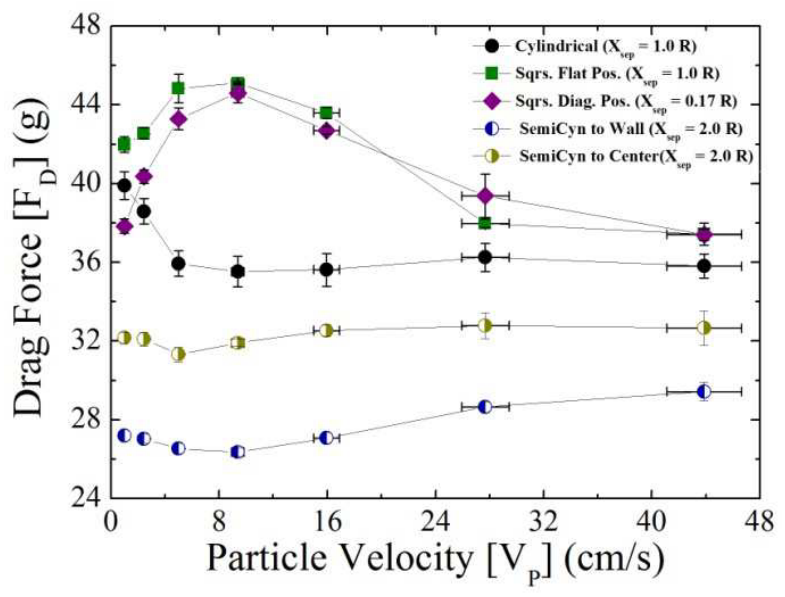

Fig. 3. Drag Force as a Function of Particle Velocity for each pair of obstacles placed in the container.

According to these results, there can be identified the following behaviours: the square obstacles (flat and inclined) have a similar behaviour between them. It can be seen that there is a maximum drag in $\mathrm{V}_{\mathrm{P}}=9.37 \pm 0.47$ $\mathrm{cm} / \mathrm{s}$ and then decreases to a value $F_{D} \sim 37 \mathrm{~g}$ for the maximum experimental particle velocity reached $\left(\mathrm{V}_{\mathrm{P}}=\right.$ $43.86 \pm 2.75 \mathrm{~cm} / \mathrm{s}$ ). When the cylindrical obstacles are placed, an inverse behaviour is observed. In this case, the plot suggests that the minimum $F_{D}$ is observed in the same value of the maximum drag for the square obstacles. Finally, the drag measured for the pair of semi-cylindrical obstacles shows a similar behaviour that the observed for the cylindrical obstacle, presenting a minimum approximately in $4.98 \pm 0.27 \mathrm{~cm} / \mathrm{s}<\mathrm{V}_{\mathrm{P}}<9.37$ $\pm 0.47 \mathrm{~cm} / \mathrm{s}$. In these cases $\mathrm{F}_{\mathrm{D}}$ (SC to Walls) $<\mathrm{F}_{\mathrm{D}}$ (SC to Centre) $<\mathrm{F}_{\mathrm{D}}$ (Cylinders). At Low velocities, in the case squares obstacles we thing that in the flat sides the probability of jamming and the arch formation is high than the other cases, because the friction between the particles and the flat face are acting along all the surface unlike in the round obstacles (complete and halves) where the friction acts in a single point. Then, when the velocity is increased; apparently the drag force for all cases trends at the same value, since the probability of jamming is decreased.

\subsection{Lift Force}

When the lift force $\mathrm{F}_{\mathrm{L}}<0$ the lift is attractive, $i$.ee. the force is towards the centre of the vessel, and when $F_{L}>$ 0 , the lift is repulsive, $i$. e. the force is towards the walls. 
The behaviour of $F_{L}$ as a function of flow velocity for each pair of obstacles can be observed in Fig. 4. For the cylindrical, square, and semi-cylindrical obstacles oriented to the centre, the lift is entirely repulsive.

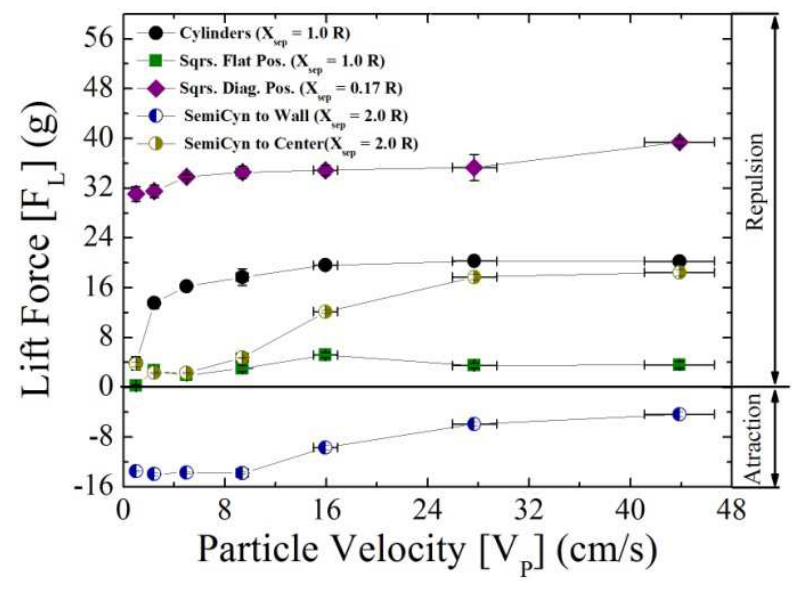

Fig. 4. Lift Force as a function of particle velocity for each pair of obstacles placed in the container.

To understand these results, it is shown in [7] that in these separations the probability of jamming is high. This implies that the quasi-static arches formed between the obstacles tends to separate the obstacles. It can be observed that the maximum lift force corresponds to the diagonal squares, which is in agreement with this hypothesis. In this case, the probability of jamming is very high since the separation is very small $\left(\mathrm{X}_{\text {sep }}=0.17\right.$ $\mathrm{R})$, and the lift is almost constant, independent of flow velocity. The cylinders have an interesting behaviour: at low velocities the lift is small, but when the flow velocity increases, the lift increases approaching asymptotically to a constant value.

The semicircles oriented towards the centre show that at low velocities the lift force is small. When the flow velocity is increased the lift force seems to reach a minimum, and then it increases and tends to values near those of the lift for cylindrical obstacles.

The squares in flat position show the lowest attractive lift force. This curve seems to confirm that the probability of jamming between the obstacles is high and thus a repulsive lift force is favoured. The position of obstacles also explains the low lift force value since in a flat position it is not easy to form a strong arc, and the few that are formed are probably formed in the edges.

Finally, the semicircles oriented towards the walls present values of lift force entirely attractive. This behaviour is consistent with the results reported by $\mathrm{Y}$. Ding et al. [2]. Interestingly, at small flow velocities the lift force is the most attractive but as the flow velocity increases the lift force tends to zero. We can speculate that at low velocity the packing fraction is high, and the granular medium exerts a larger force toward the centre. When the velocity is increased, the packing fraction is decreased, and the arcs formed between obstacles become important and they can begin to balance the force exerted by the medium. However, according this results, the arcs cannot completely balance the force from the medium and the resulting lift force is still attractive.

\section{Conclusions}

According with the results presented here we can conclude the following: 1) the drag forces are larger using faceted obstacles than rounded obstacles; and 2) the lift force is also affected by the form of the obstacles. These results suggest that attractive or repulsive forces can be altered by changing the shape of the obstacles while maintaining the global symmetry.

For future work we intend to study of the drag and lift forces varying the separation between obstacles, using the same shapes used in the present work.

\section{Acknowledgments}

M. F. Acevedo-Escalante thanks CONACYT for financial support. This work has been supported by Conacyt, Mexico, under grant 180873.

\section{References}

1. R.A. Lopez de la Cruz and G. A. CaballeroRobledo. J. Fluid Mech. 800 (2016): 248-263.

2. Y. Ding, N. Gravish, and D. I. Goldman. PRL $\mathbf{1 0 6 . 2}$ (2011): 028001.

3. F. Pacheco-Vazquez and J.C. Ruiz-Suarez. Nat. Commun. 1, 123.

4. J. M. Solano-Altamirano, G. A. Caballero-Robledo, F. Pacheco-Vazquez, V. Kamphorst and .C. RuizSuarez. PRE 88, 032206

5. D. I. Goldman. Rev. Mod. Phys. 86, 943-958.

6. I. Zuriguel, J. F. Boudet, Y. Amarouchene and H. Kellay. PRL 95, 258002

7. I. Zuriguel, L. A. Pugnaloni, A. Garcimartín and D. Maza. PRE 68, 030301.

8. Beverloo W A., Leniger H. A. and Van de Velde J. J. Chem Eng. Sci. 15, 260-296. 